\title{
ac susceptibility of the quantum critical point mimicking series $\mathrm{Li}_{x}\left[\mathrm{Mn}_{1.96} \mathrm{Li}_{0.04}\right] \mathrm{O}_{4}(x=0.0,0.1,0.2,0.35,0.5,0.6,0.8,1.0)$
}

\author{
T. Heitmann, ${ }^{1, a)}$ J. Gaddy, ${ }^{1,2}$ J. Lamsal, ${ }^{1,2}$ M. Petrovic, ${ }^{2}$ and W. Montfrooij ${ }^{1,2}$ \\ ${ }^{1}$ The Missouri Research Reactor, University of Missouri, Columbia, Missouri 65211, USA \\ ${ }^{2}$ Department of Physics and Astronomy, University of Missouri, Columbia, Missouri 65211, USA
}

(Presented 21 January 2010; received 9 November 2009; accepted 20 January 2010; published online 7 May 2010)

The present work elucidates the series of magnetic phase transitions present in the series of spinel compounds $\mathrm{Li}_{x}\left[\mathrm{Mn}_{1.96} \mathrm{Li}_{0.04}\right] \mathrm{O}_{4} \quad(x=0.0,0.1,0.2,0.35,0.5,0.6,0.8,1.0)$. These systems display dynamical scaling originating from the presence of magnetic clusters that form below $\sim 70 \mathrm{~K}$. This scaling is similar to what has been observed in the 122 quantum critical point materials containing intrinsic disorder. We study this system using ac susceptibility in order to understand how disorder leads to fragmentation of the magnetic lattice. The Li doped system's antiferromagnetic (AF) ordering sets in below $\sim 70 \mathrm{~K}$; however, for $x=1$ this ordering is limited to clusters of $\mathrm{Mn}^{4+}$ ions that are weakly coupled to each other. For the intermediate Li concentrations we observe the formation of individual spin clusters consistent with neutron scattering experiments and we find evidence for the coaligning of these clusters for $\mathrm{T} \lesssim 20 \mathrm{~K}$. A maximum in the peak of the susceptibility versus Li content between $x=0.5$ and $x=0.35$ indicates a crossover from a regime dominated by the cluster dynamics to one in which the long-range order of the delithiated $\lambda-\mathrm{MnO}_{2}$ phase begins to emerge. We discuss the magnetic phase diagram pertaining to short-range order in relationship to the dynamic response of these systems as measured by inelastic neutron scattering experiments. (C) 2010 American Institute of Physics. [doi:10.1063/1.3367976]

We present ac susceptibility measurements on the series $\mathrm{Li}_{x}\left[\mathrm{Mn}_{1.96} \mathrm{Li}_{0.04}\right] \mathrm{O}_{4} \quad(x=0.0,0.1,0.2,0.35,0.5,0.6,0.8,1.0)$. The interest in this family of materials is twofold. First, there is the practical importance owing to their use as components of light-weight batteries; ${ }^{1}$ ease of Li extraction and high voltage properties make them an attractive cathode material. Second, they have become of interest to the quantum critical point (QCP) community as they have been shown to mimic $^{2,3}$ the dynamical scaling observed in some (disordered) QCP systems. ${ }^{4-6}$ These scaling properties are believed to be directly associated with the presence of magnetic clusters similar to those observed in quantum critical systems. ${ }^{2}$ Indications of such cluster formation and freezing out of the dynamics associated with these clusters in $\mathrm{Li}_{x}\left[\mathrm{Mn}_{1.96} \mathrm{Li}_{0.04}\right] \mathrm{O}_{4}$ have been seen in neutron scattering measurements; ${ }^{7}$ however, neutrons are not able to detect the onset of cluster formation nor easily observe their freezing out. In this paper we explore this magnetic landscape of $\mathrm{Li}_{x}\left[\mathrm{Mn}_{1.96} \mathrm{Li}_{0.04}\right] \mathrm{O}_{4}$ via ac susceptibility measurements, and we show that the neutron scattering based inferences regarding the magnetic clusters are born out by our susceptibility measurements.

We briefly review what is known about short and longrange magnetism in $\mathrm{Li}_{x}\left[\mathrm{Mn}_{1.96} \mathrm{Li}_{0.04}\right] \mathrm{O}_{4}$ and in its parent compound $\mathrm{LiMn}_{2} \mathrm{O}_{4}$. The structure ${ }^{8}$ of the parent compound $\mathrm{LiMn}_{2} \mathrm{O}_{4}$, a network of eightfold $\mathrm{Mn}^{4+}$ rings surrounded by $\mathrm{Mn}^{3+}$ ions, shows that clusters are present even in nominally pure samples. Neutron scattering measurements show that this stoichiometric compound displays long-range order be-

${ }^{a)}$ Electronic mail: heitmannt@missouri.edu. low $66 \mathrm{~K} ;{ }^{9}$ the ordered pattern remains unresolved. Substitution of a small amount of $\mathrm{Li}$ ions on the $\mathrm{Mn}$ sites completely destroys this magnetic long-range order. ${ }^{10}$ Instead, neutron scattering measurements on $\mathrm{Li}_{x}\left[\mathrm{Mn}_{1.96} \mathrm{Li}_{0.04}\right] \mathrm{O}_{4}(x$ =1) show that the $\mathrm{Mn}^{4+}$ ions become correlated with each other over short distances around $\sim 70 \mathrm{~K}$, and both ${ }^{11}$ neutron scattering and muon spin relaxation measurements ${ }^{12}$ find that the dynamics associated with these $\mathrm{Mn}^{4+}$ ions freezes out below $\sim 25 \mathrm{~K}$. Neutron scattering data on the $x=0.2 \mathrm{com}-$ pound have also shown the re-emergence of long-range order at $\mathrm{T} \sim 35 \mathrm{~K}$ with the ordered pattern that of the fully delithiated phase. ${ }^{13}$ This long-range order for $x=0.2$ coexists with short-range order: neutron scattering data show magnetic Bragg peaks set upon a scattering envelope that is well described by the form factor of magnetic clusters. ${ }^{2}$ This is all the information that is known about the magnetic phase diagram as pertaining to clusters.

All measurements in this work were performed using a physical properties measurement system (PPMS) from Quantum Design. Each sample, typically $\sim 50 \mathrm{mg}$ of powder, was loaded in the PPMS and zero field cooled to $5 \mathrm{~K}$. The temperature was then ramped through the region of interest while measuring the ac susceptibility with an excitation amplitude of 15 Oe and probing frequencies of both 1 and $10 \mathrm{kHz}$. Unless otherwise noted, measurements were performed in zero applied dc field.

The resulting ac susceptibility is plotted in Fig. 1. We observe three main features: deviation from paramagnetic susceptibility near $\sim 70 \mathrm{~K}$, a well defined cusp near $\sim 20 \mathrm{~K}$, and an evolution of maximum susceptibility that peaks somewhere between $x=0.35$ and 0.5 . 

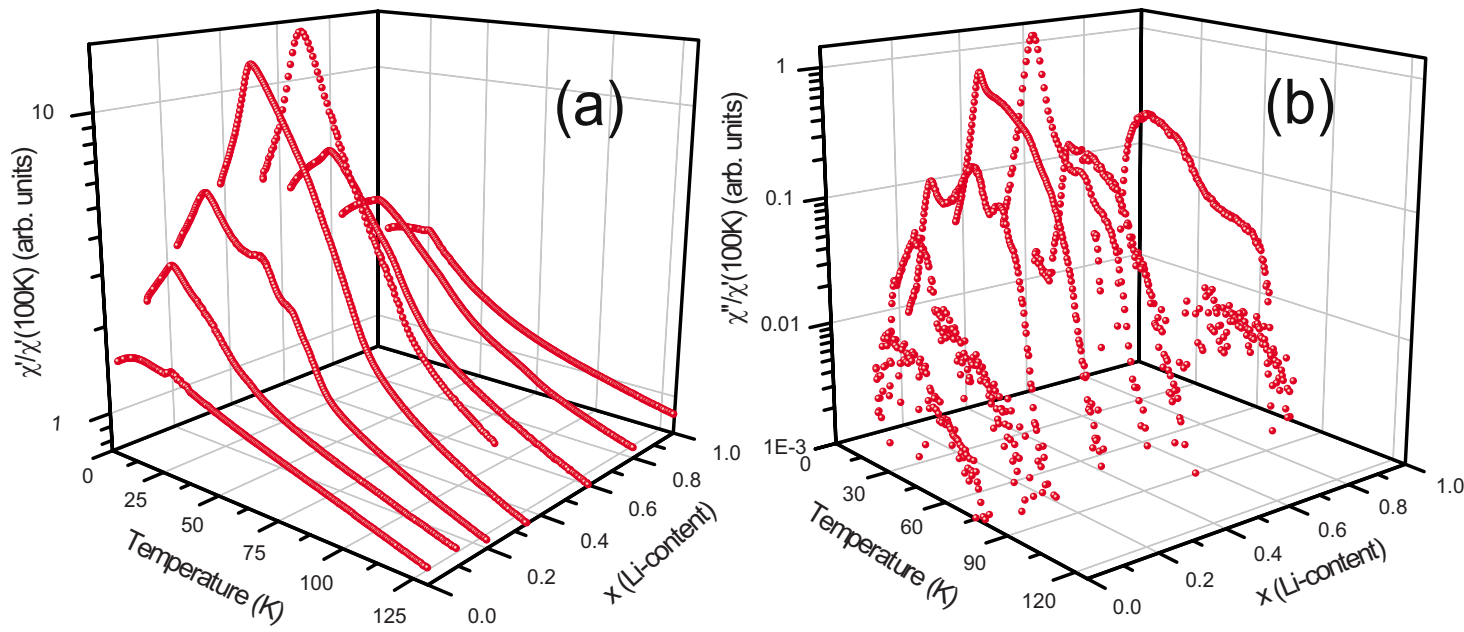

FIG. 1. (Color online) The real (a) and the imaginary parts (b) of the ac magnetic susceptibility for eight Li concentrations on a semilogarithmic scale. The data are scaled to $\chi^{\prime}(100 \mathrm{~K})$. In both graphs multiple phase transitions are observed. Note that the dissipation characterized by $\chi^{\prime \prime}$ is minimum for the $x$ $=1.0$ and 0.0 samples. The data were taking at $10 \mathrm{kHz}$ using a 15 Oe driving field.

We identify the Néel temperature $\mathrm{T}_{N}[$ Fig. 2(a)] as the temperature at which the susceptibility deviates from CurieWeiss behavior. We will take such a deviation to indicate the onset of local ordering of the $\mathrm{Mn}^{4+}$ moments. We associate this ordering temperature with the $\mathrm{Mn}^{4+}$ moments based on the unambiguous identification in neutron scattering experiments ${ }^{11}$ for the $x=1$ compound that showed that the change in dynamics at this temperature is associated with the

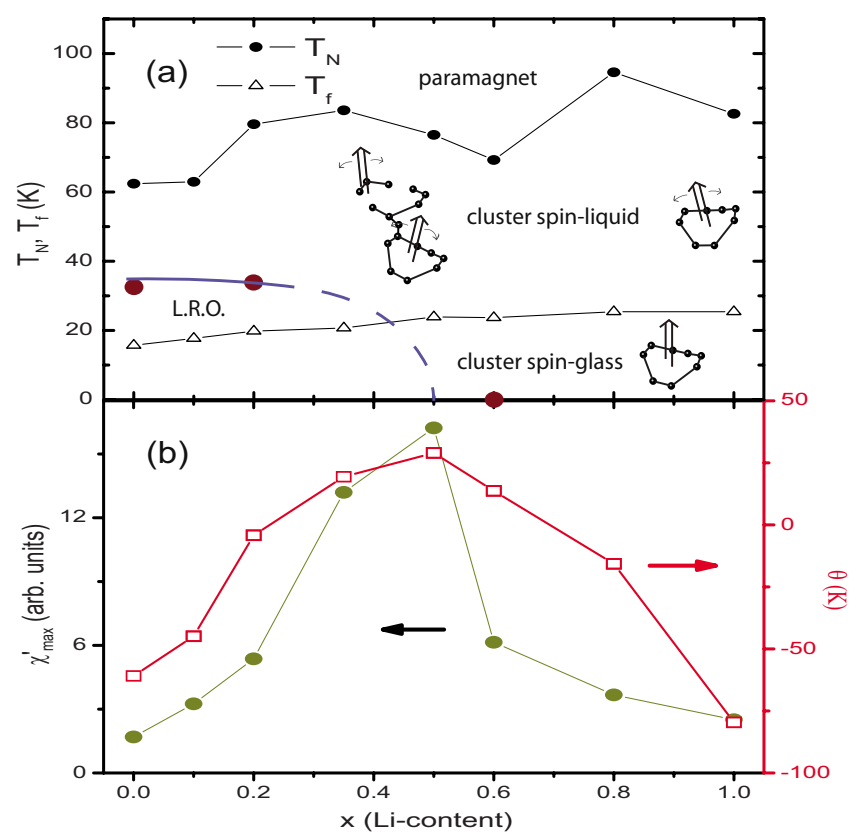

FIG. 2. (Color online) (a) The phase diagram pertaining to spin cluster formation and freezing. We use the label $\mathrm{T}_{N}$ for the temperature at which individual spins align within clusters though it should be noted that this is strictly short-range order. The inset graphics illustrate the various phases with connective single lines indicating the morphology of a cluster and the double-lined arrow indicating the cluster's AF superspin. The superspins fluctuate in the spin-liquid phase but freeze on lowering temperature into the spin glass phase region. The large dots indicate the few transitions to longrange order (LRO) that are known from the literature (Refs. 2 and 13). (b) The Weiss temperature $\theta$ (right axis) and the maximum in $\chi^{\prime}$ (left axis) are plotted as a function of $\mathrm{Li}$ content demonstrating a peak between $x=0.35$ and $x=0.5$.
$\mathrm{Mn}^{4+}$ moments, and based on the continuity of this transition temperature as a function of $x$ down to $x=0$.

We identify the predominant cusp feature in the susceptibility data near $\mathrm{T} \sim 20 \mathrm{~K}[$ Fig. $1(\mathrm{a})]$ as the freezing temperature $\mathrm{T}_{f}$ of the clusters and note that it displays only a weak dependence on Li content [see Fig. 2(a)]. Again, this identification is based on the results for the $x=1$ sample as determined from neutron scattering experiments ${ }^{11}$ and from muon spin relaxation results, ${ }^{12}$ combined with the continuity of this transition down to $x=0$. For some compositions, namely, $x=0.2$ and $x=0.35$, we observe additional transitions most likely related to the developing long-range order in those compositions, e.g., in the $x=0.2$ sample we observe as many as three distinct cusps in the data (Fig. 1). Somewhere on the delithiated end $(x<0.5)$, long-range order emerges although clustering has been shown to coexist for at least the $x=0.2$ composition. $^{2}$ For $x=0.2$, neutron scattering shows ${ }^{2}$ that long-range order sets in $(\sim 35 \mathrm{~K})$ near the lower of the two intermediate cusps [Fig. 3(d)]; therefore, the higher temperature cusp near $\sim 55 \mathrm{~K}$ must then have a different origin, perhaps ferrimagnetism from dangling moments.

Further evidence for the identification of $\mathrm{T}_{f}$ as a cluster freezing temperature comes from the frequency dependence of the susceptibility measurements. It is well established ${ }^{14}$ that for spin glass systems the freezing temperature shifts to higher temperatures with an increased probing frequency in the ac susceptibility measurement. We observe such a clear shift of the cusp near $20 \mathrm{~K}$ for the $x=0.5$ [Fig. 3(c) inset] composition. The fully lithiated sample $(x=1.0)$ displays a decidedly smaller shift with frequency. This suggests that the dynamics of the clusters are related to the dynamics of spin glass systems but that their dependence on frequency likely scales with cluster size, i.e., large clusters respond sluggishly to high frequency probing. In contrast, the delithiated samples display no dependence on frequency because of the appearance of long-range order.

We have extracted the Weiss temperatures for each composition by extrapolating the paramagnetic behavior. The results [Fig. 2(b)] show a broad peak-type feature that crosses 

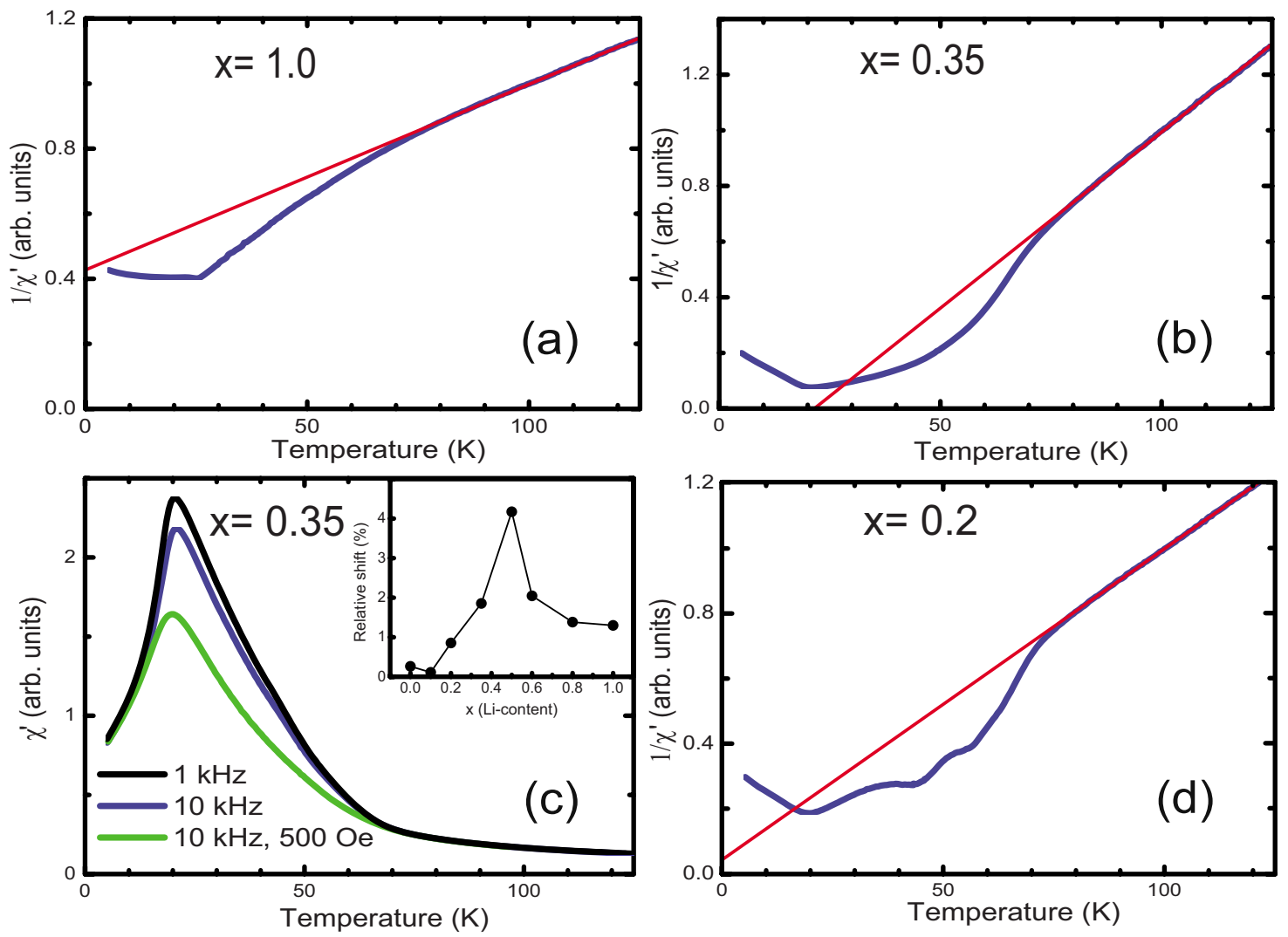

FIG. 3. (Color online) The inverse $a c$ susceptibility as a function of temperature for the fully lithiated material (a) and Li contents of $x=0.35$ (b) and 0.2 (d). Deviation from Curie-Weiss behavior is taken as an indication of local ordering. The fact that the inverse susceptibility dips below the Curie-Weiss line suggests possible ferrimagnetic behavior, weak though it may be. Part (c) shows the effect of frequency or an applied dc field on the real part of the ac susceptibility for the $x=0.35$ material and the relative shift of the freezing temperature (inset) with frequency for each composition.

to positive values for the middle of the $x$ range. We note that the peak of $\chi_{\max }^{\prime}$ with $\mathrm{Li}$ content coincides with positive Weiss temperatures.

Our susceptibility measurements combined with what has been learned from neutron scattering experiments suggests the following evolution of magnetic clusters in $\mathrm{Li}_{x}\left[\mathrm{Mn}_{1.96} \mathrm{Li}_{0.04}\right] \mathrm{O}_{4}$ as a function of $x$. On the fully lithiated end $(x=1.0)$ we find a network of magnetic clusters consisting primarily of the $\mathrm{Mn}^{4+}$ eightfold rings as described by Gaddy et $a l^{7}$ Small amounts of Li substitution on the Mn sites have disordered the neat eightfold ring pattern found ${ }^{8}$ in the stoichiometric material; the lithium ions replace some of the $\mathrm{Mn}$ ions on the $\mathrm{B}$-sites and force some of the $\mathrm{Mn}^{3+}$ ions to change to $\mathrm{Mn}^{4+}$ and partake in the $\mathrm{Mn}^{4+}$ ordering; this results in clusters that are somewhat irregular in size and shape. Removing Li from the A-sites, i.e., for $x<1$, drives more of the $\mathrm{Mn}^{3+}$ ions (surrounding the clusters) to the $\mathrm{Mn}^{4+}$ valence state. This adds moments to and creates links between the existing ring clusters, yielding a distribution of clusters that have an even or odd number of members. How many members the clusters have (on average) as a function of $x$ is as of yet unknown.

Since odd numbered clusters have a net magnetic moment, this could explain the shape of the inverse susceptibility (the dipping of $1 / \chi^{\prime}$ below the paramagnetic line shown in Fig. 3) which is indicative of ferrimagneticlike behavior. ${ }^{15}$ Interactions between neighboring clusters, mediated by $\mathrm{Mn}^{3+}$ ions, could in this case yield a ferrimagnetic behavior. Of course, it could equally well be that some of the $\mathrm{Mn}^{3+}$ are aligning with $\mathrm{Mn}^{4+}$ in the clusters to produce this effect; the size of the magnetic moments of the $\mathrm{Mn}^{3+}$ and $\mathrm{Mn}^{4+}$ ions differ so when they coalign some net moment would result as in a ferrimagnet.

This research is supported by the Department of Energy, Office of Basic Energy Sciences, Division of Materials Sciences and Engineering under Grant No. DE-FG0207ER46381.

${ }^{1}$ M. M. Thackeray, J. Am. Ceram. Soc. 82, 3347 (1999).

${ }^{2}$ T. Heitmann et al., Phys. Rev. B 81, 014411 (2010).

${ }^{3}$ J. Lamsal et al., J. Appl. Phys. 105, 07E322 (2009).

${ }^{4}$ M. C. Aronson et al., Phys. Rev. Lett. 75, 725 (1995).

${ }^{5}$ M. C. Aronson et al., Phys. Rev. Lett. 87, 197205 (2001).

${ }^{6}$ W. Montfrooij et al., Phys. Rev. Lett. 91, 087202 (2003).

${ }^{7}$ J. Gaddy et al., J. Appl. Phys. 105, 07D532 (2009).

${ }^{8}$ J. Rodríguez-Carvajal, G. Rousse, C. Masquelier, and M. Hervieu, Phys. Rev. Lett. 81, 4660 (1998).

${ }^{9}$ J. E. Greedan, C. R. Wiebe, A. S. Wills, and J. R. Stewart, Phys. Rev. B 65, $184424(2002)$

${ }^{10}$ V. W. J. Verhoeven, F. M. Mulder, and I. M. de Schepper, Physica C 276-278, 950 (2000).

${ }^{11}$ H. G. Schimmel et al., Phys. Rev. B 63, 214409 (2001).

${ }^{12}$ J. Sugiyama et al., J. Mater. Sci.: Mater. Electron. 19, 875 (2008).

${ }^{13}$ J. E. Greedan et al., Chem. Mater. 10, 3058 (1998).

${ }^{14}$ J. A. Mydosh, Spin Glasses: An Experimental Introduction (Taylor \& Francis, London, 1993).

${ }^{15}$ B. D. Cullity and C. D. Graham, Introduction to Magnetic Materials (Wiley, New Jersey, 2009). 